OPEN ACCESS

Edited by:

Qingli Dong,

University of Shanghai for Science and Technology, China

Reviewed by:

Yejun Han

University of Chinese Academy of Sciences (UCAS), China

Mariusz Cycoń,

Medical University of Silesia, Poland

*Correspondence:

Yong Zhao

yzhao@shou.edu.cn

tThese authors have contributed equally to this work.

Specialty section This article was submitted to Food Microbiology, a section of the journal Frontiers in Microbiology

Received: 08 May 2018 Accepted: 28 June 2018

Published: 26 July 2018

Citation:

Hong B, Ba Y, Niu L, Lou F, Zhang Z,

Liu H, Pan Y and Zhao Y (2018)

A Comprehensive Research on

Antibiotic Resistance Genes

in Microbiota of Aquatic Animals.

Front. Microbiol. 9:1617.

doi: 10.3389/fmicb.2018.01617

\section{A Comprehensive Research on Antibiotic Resistance Genes in Microbiota of Aquatic Animals}

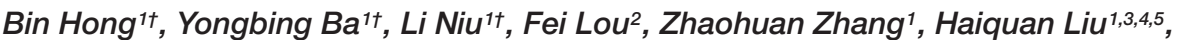 \\ Yingjie Pan ${ }^{1,3,4}$ and Yong Zhao ${ }^{1,3,4 *}$
}

${ }^{1}$ College of Food Science and Technology, Shanghai Ocean University, Shanghai, China, ${ }^{2}$ Agri-Products Quality and Safety Testing Center of Shanghai, Shanghai, China, ${ }^{3}$ Shanghai Engineering Research Center of Aquatic-Product Processing and Preservation, Shanghai, China, ${ }^{4}$ Laboratory of Quality and Safety Risk Assessment for Aquatic Products on Storage and Preservation (Shanghai), Ministry of Agriculture, Shanghai, China, ${ }^{5}$ Engineering Research Center of Food

Thermal-Processing Technology, Shanghai Ocean University, Shanghai, China

The occurrence of antibiotic resistance genes (ARGs) as emerging contaminants is of continued concern for human health. Antibiotics used in aquaculture have promoted the evolution and spread of ARGs. This study aimed to investigate the occurrence of 37 ARGs conferring resistance to six classes of antibiotics in 94 aquatic animals from five cities in southeast coast of China. The results showed that floR, sulll, sull, strB, str $\mathrm{A}$, aadA, and tetS were identified as the prominent ARGs with the high detection frequencies ranging from 30.9 to $51.1 \%$ in total samples. Then relative expression amount of seven prominent ARGs quantified by qPCR, ranging from 0.003 to 0.065 . The tetS was the most abundant ARG among the seven ARGs. Though aadA was the second highest detection frequency of ARGs, it was the lowest expression amount ARG. The occurrences and abundances of ARGs in freshwater aquatic animals were greater than those in marine, reflecting the discrepancy of cultivation pattern between the freshwater and marine aquaculture. Shanghai was considered as the most prevalent site with 16 ARGs, and Ningbo merely contained 9 ARGs without of $\beta$-lactam ARGs and quinolone ARGs, showing variations of ARGs with geographical location. Eight kinds of sulfonamides and one chloramphenicol residues were further measured in samples from Shanghai. Interestingly, no target antibiotics were found, but sulfonamides resistance genes (sull, sulll) and chloramphenicol resistance genes (floR) persisted at aquatic animals in the absence of selection pressure. Our research firstly shows comprehensive information on the ARGs in skin microbiota of aquatic animals, which could provide useful information and a new insight for better understanding on the ARGs dissemination in aquatic animals.

Keywords: aquatic animals, antibiotic resistance genes, skin microbiota, southeast coast of China, sulfonamides, chloramphenicol

\section{INTRODUCTION}

Antibiotic resistance genes (ARGs), emerging environmental contaminants, draw an increasingly attention due to their huge risk to human health (Pruden et al., 2006). ARGs encoding resistance to a broad range of antibiotics have been found to be able to spread among bacteria via horizontal gene transfer (HGT), thus aggravating ARGs dissemination (Thomas and Nielsen, 2005; 
Bellanger et al., 2014). In addition, antibiotic-resistance bacteria that are associated with wild animals is correlated with the proximity of the animals (and the bacteria) to human populations. Migratory animals are important contributors to the widespread dissemination of ARGs (Allen et al., 2010). Many bacteria, especially human commensal bacteria and pathogens, have been reported to be multi-drug resistant (Hatha et al., 2005) and capable of transferring their resistance determinants among environmental bacteria of different genera (Agersø and Petersen, 2007; Guglielmetti et al., 2010), which results in a huge adverse effect to human health. Also, the World Health Organization (WHO) pointed out that antibiotic resistance already had become one of the greatest threats to aquatic food and, consequently, global public health through the food chain $^{1}$.

In 2016 , the total number of aquatic products was nearly 70 million tons in China, an increase of $3.69 \%$ than last year, of which aquaculture products accounted for 73\% (CAP, 2016). To sustain the rapid and steady growth of this industry, it's necessary to obtain the profit of aquaculture by using antibiotics to ensure high animal growth and low infectious diseases. The broad-spectrum antibiotics had been vastly used in aquaculture and husbandry since they were found that they could be used not only for the prevention and treatment of infectious diseases, but also for promotion of animal growth and feed efficiency (Gustafson and Bowen, 1997). In China, the annual usage of raw antibiotic ingredients for both human and agriculture is up to $180,000 \mathrm{t}$ (Zheng et al., 2012). Approximately $70 \%$ of these antibiotics are excreted in the unaltered forms, which are eventually discharged into the water environment in a variety of ways, such as through the disposal of sewage, hospital wastewater and animal waste (Kümmerer and Henninger, 2003; Yang et al., 2011). All these scenarios and the artificially added antibiotics posed a great threat to both freshwater and marine aquaculture.

Southeast coast of China, typical subtropical climate, is one of the fastest developing and most highly urbanized regions in China. However, to our knowledge, most studies to date have focused on antibiotic contamination in one area alone, like Shanghai, Hangzhou, Ningbo, Fuzhou, and Xiamen (Jiang et al., 2011; Li et al., 2011; Ji-Bing and Hu, 2012; Shen et al., 2014; Tang et al., 2017), very few comprehensive researches on ARGs in microbiota of aquatic animals have been paid attention to in these area together (Xu et al., 2007). In addition, many reports were available on the occurrence and abundance of ARGs and antibiotic residues in water and sediment in aquaculture environment (Gao et al., 2012; Cheng et al., 2013; Chen et al., 2017; Nakayama et al., 2017). However, there are very few reports on ARGs and antibiotic residues in aquatic animals, therefore, it is necessary to investigate ARGs and antibiotic residues in aquatic animals of this area.

Compared to many previous researches on aquatic animal gut microbiota (Xiong et al., 2015; Muziasari et al., 2016b; Fu et al., 2017), to the best of our knowledge, this is a first comprehensive study on antibiotics in aquatic animal skin

${ }^{1}$ http://www.who.int/mediacentre/factsheets/antibiotic-resistance/en/ microbiota. The purpose of this study aimed to investigate the occurrence of 37 ARGs conferring resistance to six classes of antibiotics in 94 aquatic animals from five cities (Shanghai, Hangzhou, Ningbo, Fuzhou, Xiamen) located in southeast coastal area of China. Eight kinds of sulfonamides and one chloramphenicol residues were further measured in samples from Shanghai. This investigation would give us a new insight on the ARGs dissemination in aquatic animals, which might be paid attention to.

\section{MATERIALS AND METHODS}

\section{Sample Sites and Sample Collection}

Sixteen species of 94 aquatic animals were collected in August to October 2015 from five southeast coastal cities of China including Shanghai, Hangzhou, Ningbo, Fuzhou, Xiamen. The samples were obtained from local markets. After sampling, they were stored at $-80^{\circ} \mathrm{C}$ for further analysis. A summary of the sampling and reference sites is shown in Table 1.

\section{DNA Extraction}

All aquatic animals were dissected under aseptic conditions. The DNA of skin bacteria was extracted by using TIANamp Bacteria DNA Kit (Tiangen Biotech Beijing Co., Ltd., China) according to the manufacturer's instructions. There was a slight modification in extraction method, where the incubation time in lysozyme was increased to $1 \mathrm{~h}$ and the incubation time in proteinase $\mathrm{K}$ was increased to $2 \mathrm{~h}$ (Ye et al., 2013). The quality of extracted DNA was verified by $2 \%$ agarose gel electrophoresis, and the concentrations of DNA were measured by multifunction enzyme labeling instrument (BioTek Synergy2, American). At last, the DNA was stored at $-20^{\circ} \mathrm{C}$ prior to PCR analysis.

\section{Detection of ARGs}

The presence of 37 ARGs were identified by PCR, including $\beta$-lactam ARGs (CARB, SHV, SHV-5, ampC, mecA), tetracycline $\mathrm{ARG}$ (tet $\mathrm{A}, \operatorname{tet} \mathrm{B}, \operatorname{tet} \mathrm{M}, \operatorname{tet} \mathrm{O}$, tet $\mathrm{Q}, \operatorname{tet} \mathrm{S}$, tet $\mathrm{W}$, tet $\mathrm{K})$, aminoglycoside ARGs (aph $\left(2^{\prime}\right)-I b, s t r \mathrm{~A}, \operatorname{str} \mathrm{B}, \operatorname{aad\mathrm {A}}, \operatorname{aad\mathrm {E}}$ $\left.\operatorname{aac}\left(6^{\prime}\right)-\mathrm{Ib}, \operatorname{arm\mathrm {A}}, \mathrm{rmt \textrm {B }}\right)$, quinolone ARGs ( $q n r \mathrm{~S}, \operatorname{aac}\left(6^{\prime}\right)-\mathrm{Ib}-\mathrm{cr}$, $q n r \mathrm{~A}, q n r \mathrm{C}, q n r \mathrm{D}$, parC, $q n r \mathrm{~B})$, chloramphenicol ARGs (cat $\mathrm{I}$, cat II, cat III, catIV, floR), and sulfonamide ARGs (sulI, sulII, sulIII, sulA). Primers of all target ARGs (Supplementary Table S1) were synthesized based on our previous study (Lou et al., 2015; Li et al., 2017). PCR results were sequenced by Sangon Biotech (Sangon Biotech, Shanghai, China) and analyzed by the National Center for Biotechnology Information website $(\mathrm{NCBI})^{2}$.

\section{Quantification of 16S rRNA and ARGs}

Identified prominent ARGs (tetS, strA, strB, aadA, sulI, and sulII) were further quantified by using SYBR Green quantitative

\footnotetext{
${ }^{2}$ http://www.ncbi.nlm.nih.gov/BLAST
} 
TABLE 1 | The sites and species of 94 samples collected in this study.

\begin{tabular}{|c|c|c|c|c|c|c|c|}
\hline \multicolumn{2}{|c|}{ Species } & \multicolumn{6}{|c|}{ The number of samples } \\
\hline & & Shanghai & Hangzhou & Ningbo & Fuzhou & Xiamen & Total \\
\hline \multirow[t]{8}{*}{ Freshwater aquatic animals (62) } & Largemouth bass (Micropterus salmoides) & 3 & 3 & 2 & 1 & 3 & 12 \\
\hline & Bighead carp (Hypophthalmichthys nobilis) & 1 & 1 & 3 & 2 & 1 & 8 \\
\hline & Common carp (Cyprinus carpio) & 2 & - & - & - & - & 2 \\
\hline & Mandarin fish (Siniperca chuatsi) & 3 & 2 & 1 & 1 & 1 & 8 \\
\hline & Crucian carp (Carassius carassius) & 8 & 2 & 1 & 3 & 1 & 15 \\
\hline & Snakehead (Channa argus) & 3 & 1 & 1 & - & - & 5 \\
\hline & Yellowhead catfish (Pelteobagrus fulvidraco) & 2 & 1 & - & 1 & - & 4 \\
\hline & Grass carp (Ctenopharyngodon idella) & 2 & 2 & 2 & 2 & - & 8 \\
\hline \multirow[t]{8}{*}{ Marine aquatic animals (32) } & Turbot (Scophthalmus maximus) & 4 & 1 & 1 & 1 & 1 & 8 \\
\hline & Pompano (Trachinotus ovatus) & - & - & - & - & 3 & 3 \\
\hline & Large yellow croaker (Larimichthys crocea) & 3 & 1 & - & - & - & 4 \\
\hline & Yellow grouper (Epinephelus awoara) & - & - & 2 & 2 & 3 & 7 \\
\hline & Japanese sea bream (Pagrus major) & - & - & 2 & - & 1 & 3 \\
\hline & Red drum (Sciaenops ocellatus) & - & - & - & 1 & 1 & 2 \\
\hline & Pacific white shrimp (Litopenaeus vannamei) & 1 & 1 & - & 1 & - & 3 \\
\hline & Giant tiger prawn (Penaeus monodon) & 1 & - & - & 1 & - & 2 \\
\hline Total & & 33 & 15 & 15 & 16 & 15 & 94 \\
\hline
\end{tabular}

real-time PCR (qPCR). The $\mathrm{qPCR}$ assays were performed on ABI7500 (Applied Biosystems, United States), and the reaction system $(20 \mu \mathrm{L})$ included $10 \mu \mathrm{L}$ SYBR Green Premix (Sangon Biotech, Shanghai, China), $0.3 \mu \mathrm{L}$ of each primer and $2 \mu \mathrm{L}$ of template DNA. The detail qPCR program was as follows: $1 \mathrm{~min}$ at $95^{\circ} \mathrm{C}$, followed by 40 cycles of $15 \mathrm{~s}$ at $95^{\circ} \mathrm{C}, 30 \mathrm{~s}$ at annealing temperature (Supplementary Table S2), $30 \mathrm{~s}$ at $72^{\circ} \mathrm{C}$. Resistance genes (tet $\mathrm{S}, \operatorname{str} \mathrm{A}, \operatorname{str} \mathrm{B}, \operatorname{aad} \mathrm{A}, s u l \mathrm{I}$, and $s u l \mathrm{II})$ and reference gene (16S rDNA) were cloned into TA vector and then transfected into Escherichia coli DH5 $\alpha$ (Tiangen Biotech Beijing Co., Ltd., China). Plasmids carrying target genes were used to generate calibration curves, and negative controls were performed for each run. The qPCR efficiencies ranged from 90 to $110 \%$ with $R^{2}$-values greater than 0.99 for all calibration curves, and the analytical conditions were described in previous study (Chen et al., 2017).

\section{Antibiotic Residues Analysis}

The antibiotic standards, including eight kinds of sulfonamides (sulfadiazine, sulfathiazole, sulfamethyldiazine, sulfamethazine, sulfamethoxazole, sulfadoxine, sulfisoxazole, and sulfaquinoxaline) and chloramphenicol, were obtained from ALADDIN Chemical Co., Ltd. (Shanghai, China). The antibiotic solutions were prepared at a concentration of $500 \mu \mathrm{g} / \mathrm{ml}$ in methyl alcohol and stored at $-80^{\circ} \mathrm{C}$ for further experiment.

Thirty-three aquatic samples from Shanghai were selected to analyze antibiotic residues. All aquatic animals were dissected under aseptic conditions and muscles were used to detect antibiotic residues. Ten grams of aquatic animals were selected, and the experimental samples were placed in $50 \mathrm{ml}$ centrifuge tubes and extracted by Mellvmince-EDTA buffer. The concentration of target antibiotics in aquatic animals was measured by high performance liquid chromatographytandem mass spectrometry (HPLC-MS/MS) system (Manchester,
United Kingdom). The analytical conditions were described in previous study (Hon et al., 2016).

\section{Data Analysis}

The occurrences and abundances of ARGs were performed with OriginPro 9.1 (OriginLab, United States). The average values were calculated by Excel 2016 (Microsoft, United States), Heatmap analysis was conducted by HemI version 1.0 (Heatmap Illustrator, China).

\section{RESULTS}

\section{ARGs Diversity in Aquatic Animals}

Nineteen of thirty-seven target ARGs were found with detection frequencies ranging from 2.1 to $51.1 \%$ in total aquatic animals (CARB, SHV, SHV-5, mecA, tet $\mathrm{A}$, tet $\mathrm{S}$, tet $\mathrm{K}, \operatorname{str} \mathrm{A}, \operatorname{str} \mathrm{B}, \operatorname{aadA}$, $a a d \mathrm{E}, a a c\left(6^{\prime}\right)-I b, \operatorname{armA}, a a c\left(6^{\prime}\right)-I b-c r, q n r \mathrm{~A}, q n r \mathrm{D}$, flo R, sulI, and sulII) (Figure 1). The flo R, sulII, sulI, strB, str A, aad A, and tet $\mathrm{S}$ were identified as the prominent ARGs with the high detection frequencies ranging from 30.9 to $51.1 \%$, suggesting a serious condition conferring resistance to tetracyclines, aminoglycosides, chloramphenicol, and sulfonamides in aquatic animals. All categories were detected in the six categories ARGs. For $\beta$-lactam ARGs, four encoding $\beta$-lactamase genes, CARB, SHV, SHV-5 and $m e c A$, were detected with low detection frequencies ranging from 3.2 to $20.2 \%$. Three (tet $\mathrm{A}$, tet $\mathrm{S}$, tet $\mathrm{K}$ ) of eight tetracycline ARGs were found with the detection frequency ranging from 8.5 to $51.1 \%$. It is noteworthy that tet $\mathrm{S}$ exhibited the highest frequently detection of the 37 ARGs in our study $(51.1 \%, n=94)$. Six of eight aminoglycoside ARGs were found ranging from 2.1 to $47.9 \%$. The $\mathrm{q} n r \mathrm{D}$ was the predominant ARG detected in seven quinolone ARGs, with the detection frequency of $27.7 \%$, followed 


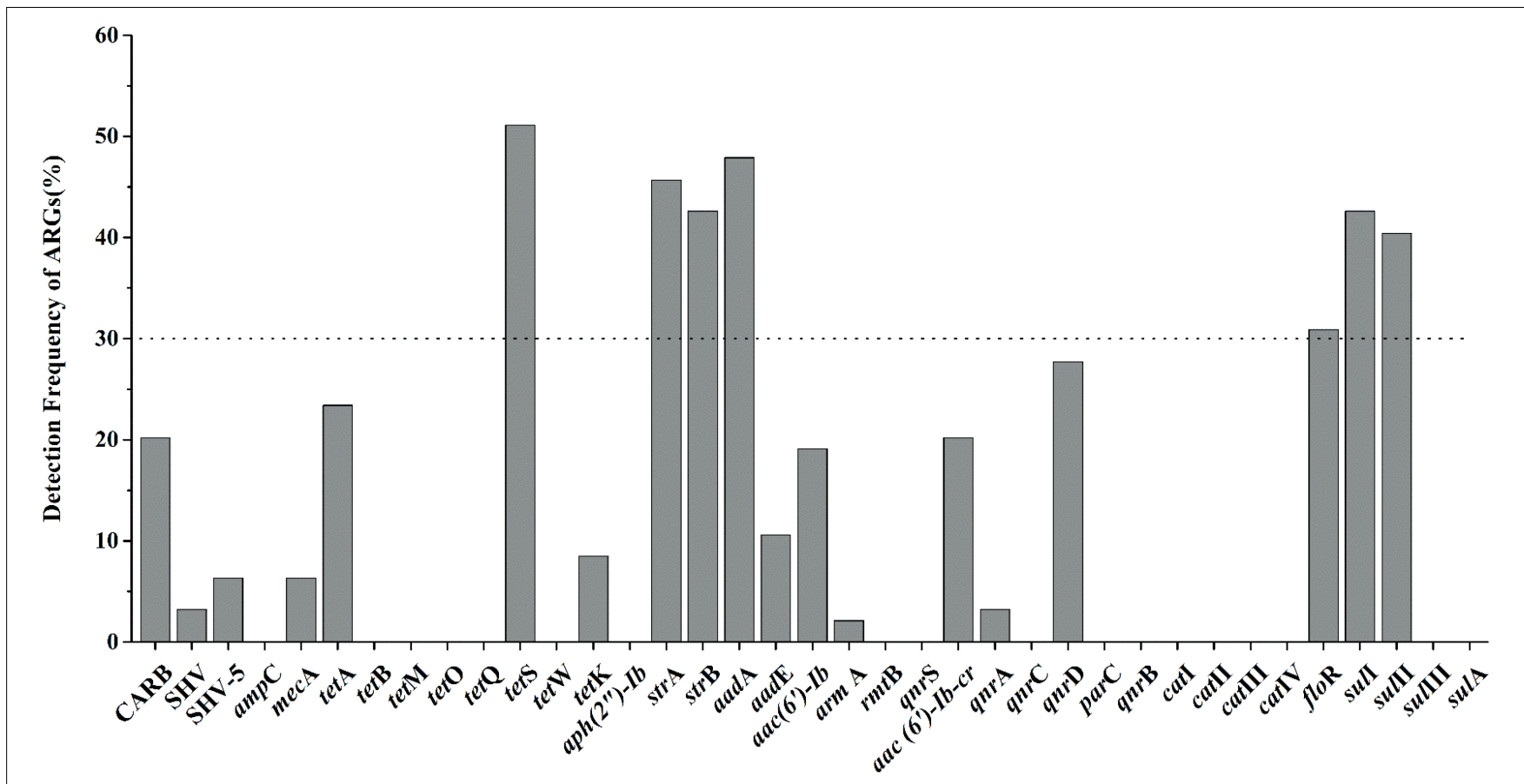

FIGURE 1 | The frequencies of 37 antibiotic resistance genes (ARGs) conferring resistance to six classes of antibiotics in total aquatic animals. (The dotted line shows seven major genes with a detection rate of more than $30 \%)$.

by $\operatorname{aac}\left(6^{\prime}\right)-\mathrm{Ib}-\mathrm{cr}$ and qnrA (20.2 and 3.2\%). The floR was the only chloramphenicol ARGs with the high detection frequency $(30.9 \%, n=94)$. In sulfonamides ARGs, sulI, and sulII exhibited the high detection frequencies of 41.5 and $40.4 \%$, but sulIII and sulA were not observed in any samples.

\section{The Seven Prominent ARGs Relative Expression Amount Analysis}

The average relative expression (to internal reference gene $16 \mathrm{~S}$ rDNA) of seven prominent ARGs in total aquatic animals decreased as: tet $\mathrm{S}>\operatorname{str} \mathrm{A}>\operatorname{str} \mathrm{B}>$ sul $>$ sul $\mathrm{II}>$ flo $\mathrm{R}>$ aad $\mathrm{A}$, ranging from 0.003 to 0.065 (Figure 2). The tet $\mathrm{S}$ was the highest abundant ARG in aquatic animals among the seven prominent ARGs, with the average relative expression in tet $\mathrm{S}$ positive samples $1.27 \times 10^{-1} / 16 \mathrm{~S} \mathrm{rDNA}$ (the relative abundance ranged from $2.00 \times 10^{-4}$ to $9.46 \times 10^{-1} / 16 \mathrm{~S} \mathrm{rDNA}, n=48$ ). Slight gaps were observed in the average relative expression of str $\mathrm{B}$, flo R, sulI, and sulII. Though aadA was the second highest detection frequency of 37 ARGs, it was the lowest abundant ARG. The average relative expression in aadA positive samples was $6.30 \times 10^{-3} / 16 \mathrm{~S}$ rDNA $(n=45)$. Overall, tetS was also the highest frequently detection of 37 ARGs.

\section{Comparison ARGs Diversity Between Freshwater and Marine Aquatic Animals}

Eighteen of thirty-seven target ARGs were found in freshwater aquatic animals with detection frequencies ranging from 3.2 to $59.7 \%$, also, 18 genes were found in marine aquatic animals with detection frequencies ranging from 3.1 to $43.8 \%$ (Figure 3 ). The

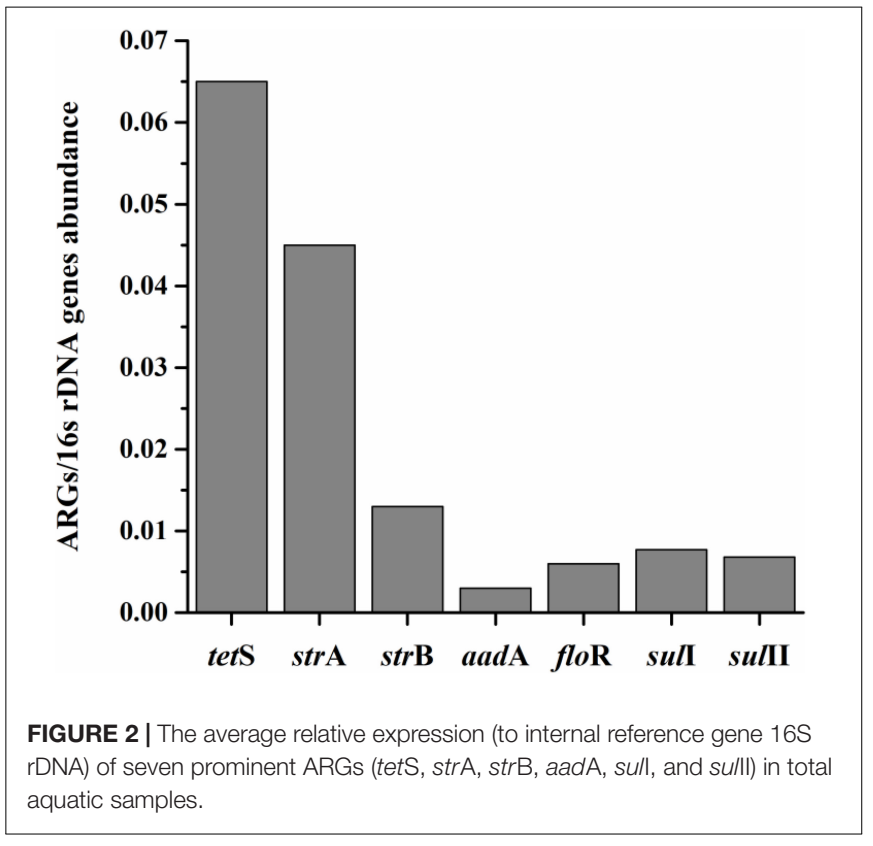

tetS was the highest detection of the 37 ARGs in freshwater aquatic animals $(59.7 \%, n=62)$, but the aadA was the highest detection of the 37 ARGs in marine aquatic animals (43.8\%, $n=32)$. Interestingly, the SHV $(4.8 \%, n=62)$ of $\beta$-lactam ARGs was the only one existed in freshwater aquatic animals, and the $\operatorname{armA}(6.3 \%, n=32)$ of aminoglycoside ARGs was the only one existed in marine aquatic animals. 


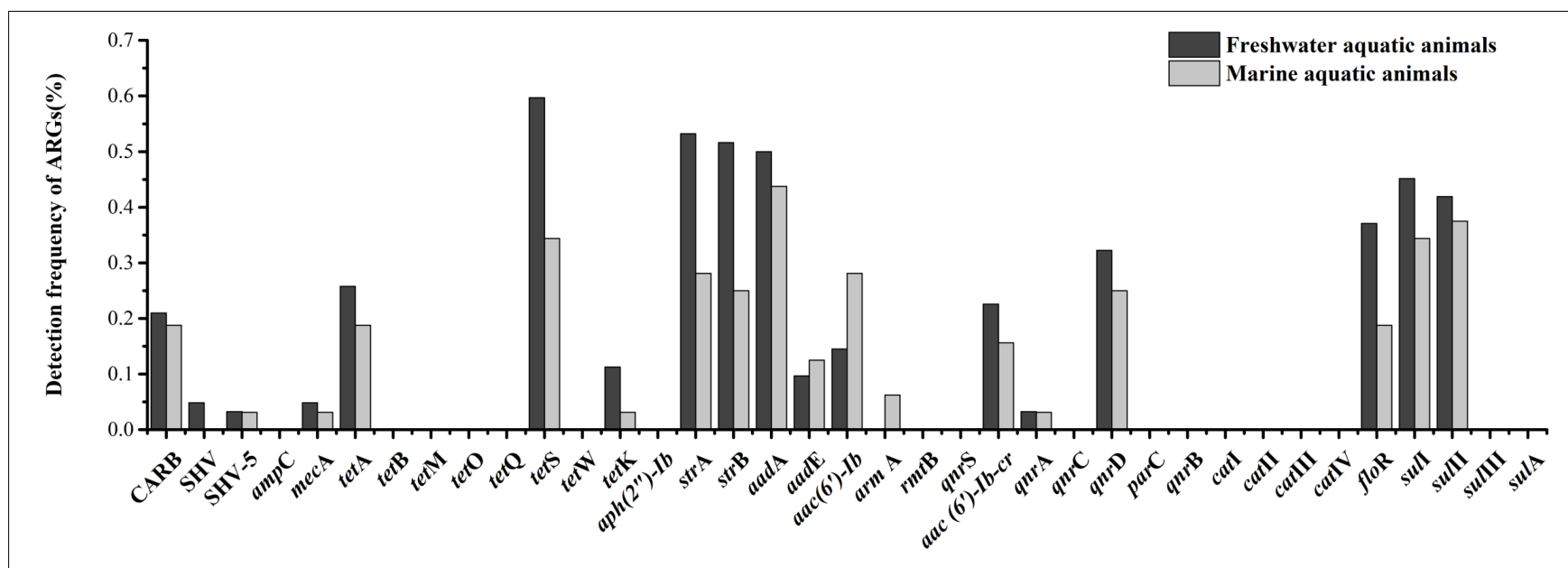

FIGURE 3 | The frequencies of 37 ARGs detected in freshwater aquatic animals and marine aquatic animals.

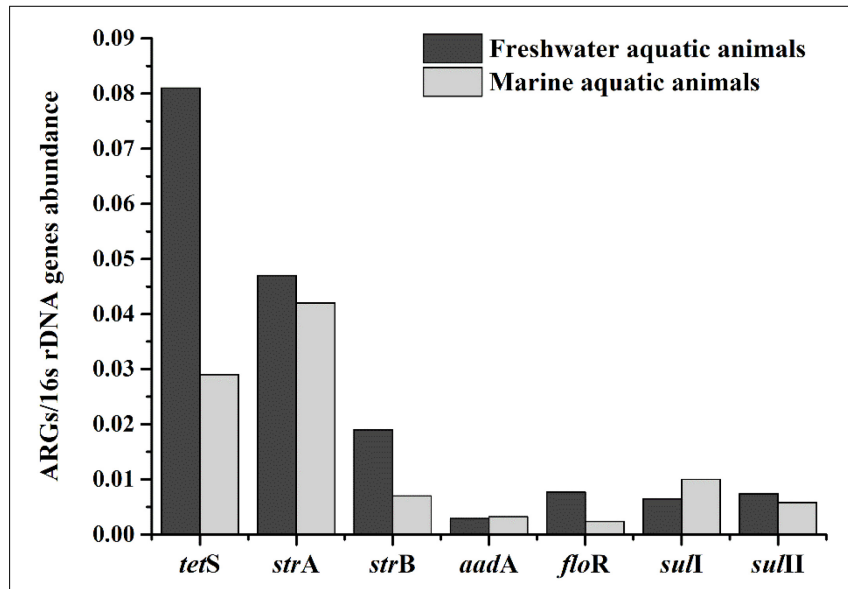

FIGURE 4 | The average relative expression of seven prominent ARGs (tetS, strA, strB, aadA, sull, and sulll) in freshwater aquatic animals and marine aquatic animals.

The relative expression amount of seven prominent ARGs in freshwater aquatic animals also were higher than those in marine aquatic animals (Figure 4). The average relative abundance of freshwater aquatic animals was 0.025 , being approximately 1.73 times greater than those in marine aquatic animals. The tet $\mathrm{S}$ was the highest abundant ARG in freshwater aquatic animals, but the strA was the highest abundant ARG in marine aquatic animals. Overall, the occurrences and abundances of ARGs in freshwater aquatic animals were greater than those in marine aquatic animals.

\section{ARGs Diversity With Geographical Location}

Considering ARGs presence in different cities (Figure 5), Shanghai was considered the most prevalent site with sixteen ARGs (CARB, SHV, mecA, tet A, tet $\mathrm{S}$, tet $\mathrm{K}$, str A, strB, aadA, aadE, $a a c\left(6^{\prime}\right)-I b, a a c\left(6^{\prime}\right)-I b-c r, q n r \mathrm{D}, f l o \mathrm{R}, s u l \mathrm{I}$, and $\left.s u l \mathrm{II}\right)$, whereas
Ningbo contained nine ARGs (tetS, strA, strB, aadA, aac $\left(6^{\prime}\right)$ Ib, armA, floR, sulI, and sulII), with only nine ARGs and none of $\beta$-lactam ARGs and quinolone ARGs, showing variations of ARGs with geographical location (Figure 6). All the prominent seven resistance genes tet $\mathrm{S}, \operatorname{str} \mathrm{A}, \operatorname{str} \mathrm{B}, \operatorname{aad\mathrm {A}}$, flo $\mathrm{R}, \operatorname{sul\mathrm {I}}$, and $s u l \mathrm{II}$ were observed in five cities samples.

The average relative expression of tet $\mathrm{S}$ was the most abundant ARG in Shanghai (0.066), Fuzhou (0.13), and Xiamen (0.038), but the strA was the most abundant ARG in Hangzhou (0.11) and Ningbo (0.11). The total concentration of seven ARGs in Hangzhou is the highest abundant in five cities followed by Fuzhou, Shanghai, Ningbo, and Xiamen (Figure 7). This result reflected a fact that aquatic animals in the southeast coastal area of China contained various ARGs, furthermore, tetS, strA, strB, aadA, floR, sulI, and sulII posed a leading position in ARG contaminations.

\section{Relationship Between Antibiotic Residues and ARGs}

Shanghai was considered as the most prevalent site with 16 ARGs in five cities, so thirty-three samples from Shanghai were chosen to analyze antibiotic residues, including sulfadiazine, sulfathiazole, sulfamethyldiazine, sulfamethazine, sulfamethoxazole, sulfadoxine, sulfisoxazole, sulfaquinoxaline, and chloramphenicol. Though chloramphenicol ARGs (floR) and sulfonamide ARGs (sulI and sulII) were dominant ARGs in aquatic animals in our study, it was worth noting that no target antibiotics were detected (the detection limit of target antibiotics were $1.0 \mu \mathrm{g} / \mathrm{kg}$ ). Result showed that floR, sulI, and sulII persist at aquatic animals in the absence of selection pressure (Table 2 ).

\section{DISCUSSION}

Antibiotic resistance genes can pose a threat to food security and human health via various pathways (Cabello, 2004; Liu et al., 2014; Aydin et al., 2015; Amandine et al., 2016). Most ARGs acquired through HGT had been originated in environmental 


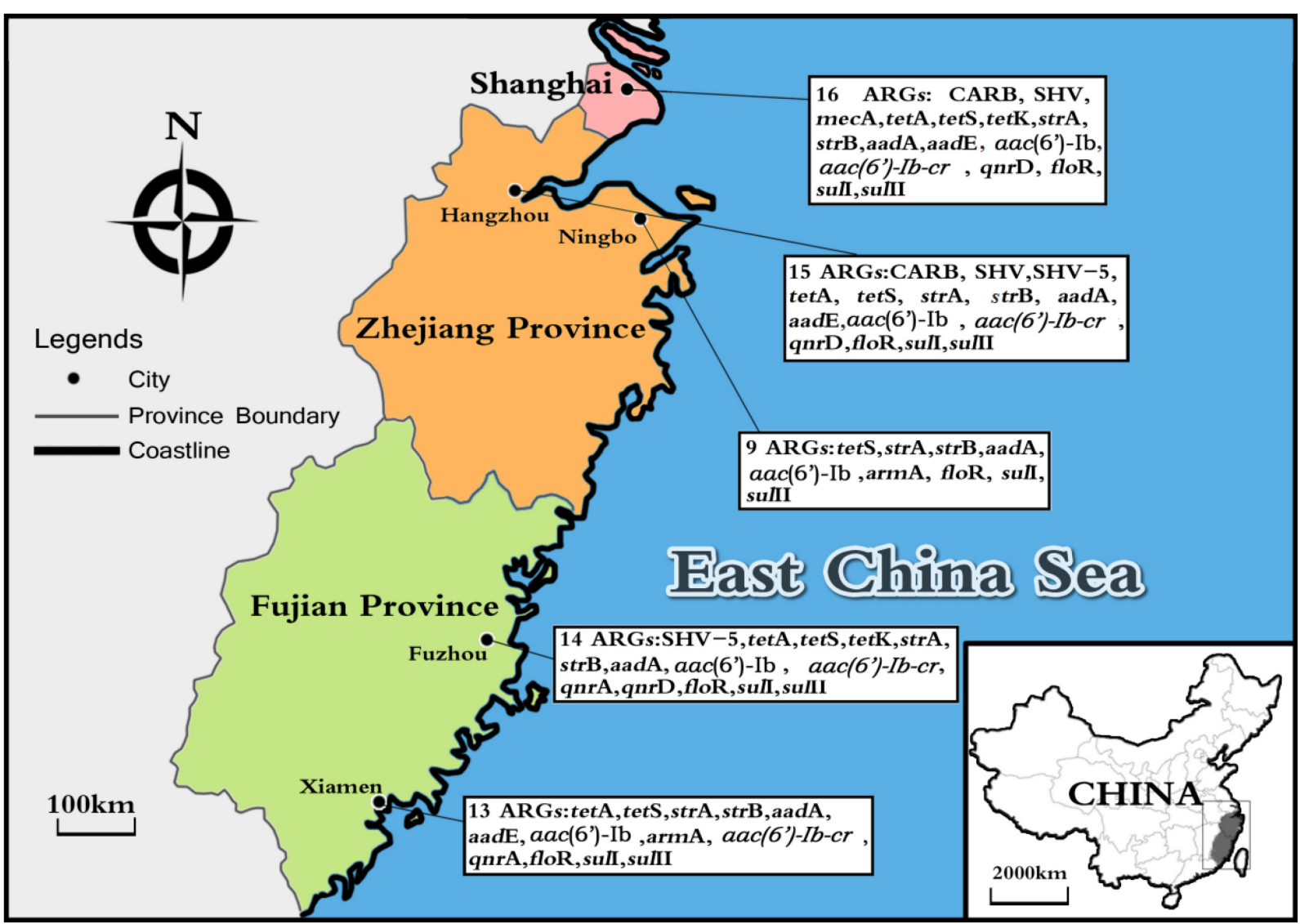

FIGURE 5 | The sites of total aquatic animals collected in five southeast coastal cities of China (Shanghai, Hangzhou, Ningbo, Fuzhou, and Xiamen) and ARGs diversity distribution.

microbiota (Martínez, 2008; Wang et al., 2012), and ARGs disseminated from farming source to reared organisms ( $\mathrm{Su}$ et al., 2017). In addition, many previous studies mainly focused on sediments, water and aquatic animal gut microbiota (Khan et al., 2013; Fu et al., 2017; Gao et al., 2018), this study firstly reflected the occurrence of 37 ARGs conferring resistance to six classes of antibiotics in skin microbiota of aquatic animals from southeast coastal area of China, which provides a comprehensive profile on ARGs in microbiota of aquatic animals.

In our study, occurrence of 37 ARGs were investigated in 94 aquatic animals from five cities in southeast coast of China. The tet $\mathrm{A}$, tet $\mathrm{K}$, and tet $\mathrm{S}$ were found with the detection frequencies ranging from 8.5 to $51.1 \%$, since wide usage of tetracyclines in environment might address this case (Cheesanford et al., 2001; Heuer et al., 2009; Yan et al., 2018a,b). In addition, the str A, strB, sulI, and sulII were prevalent in this study, which was consistent with the previous findings about the occurrence of ARGs obtained from aquaculture sediment and aquaculture water (Gao et al., 2012; Xiong et al., 2015; Muziasari et al., 2016a), and also similar to previous reports in swine farms, broiler feedlots and domestic sewage (Zhu et al., 2013; Chen et al., 2016; He et al., 2017). Results suggest that antibiotic usage pose a serious threat to the aquaculture and water environment, consequently, to public health through the food chain. The tet $\mathrm{B}$, tet $\mathrm{M}$, tet $\mathrm{O}$, tet $\mathrm{Q}$, and tet $\mathrm{W}$ were negative, this result is different from those observed in aquaculture farms, where the tet $\mathrm{M}$, tet $\mathrm{W}$, tet $\mathrm{Q}$, tet $\mathrm{O}$ were found in aquaculture farm sediments and water (Ma et al., 2014; Su et al., 2014). The occurrence of ARGs obtained from aquatic animal skin microbiota is inconsistent with the sediments, water and aquatic animal gut (Khan et al., 2013; Fu et al., 2017; Gao et al., 2018). In this study, aquatic animal skin microbiota may be another important niche for dissemination of ARGs. And the mobility of aquatic animal may facilitate the proliferation and propagation of ARGs in water environment.

A previous study have investigated the heavy metal in marine aquatic animal and freshwater aquatic animal in south China (Cheung et al., 2008), however, no study focused on ARGs differences between freshwater aquatic animals and marine aquatic animals. Results showed that occurrences and abundances of ARGs in freshwater animals were greater than those in marine aquatic animals, reflecting the importance of freshwater aquaculture environment in ARGs emergence. The discrepancy of cultivation pattern might address this phenomenon. Compared to marine aquatic animals, aquatic animals in freshwater aquaculture environment could easily 


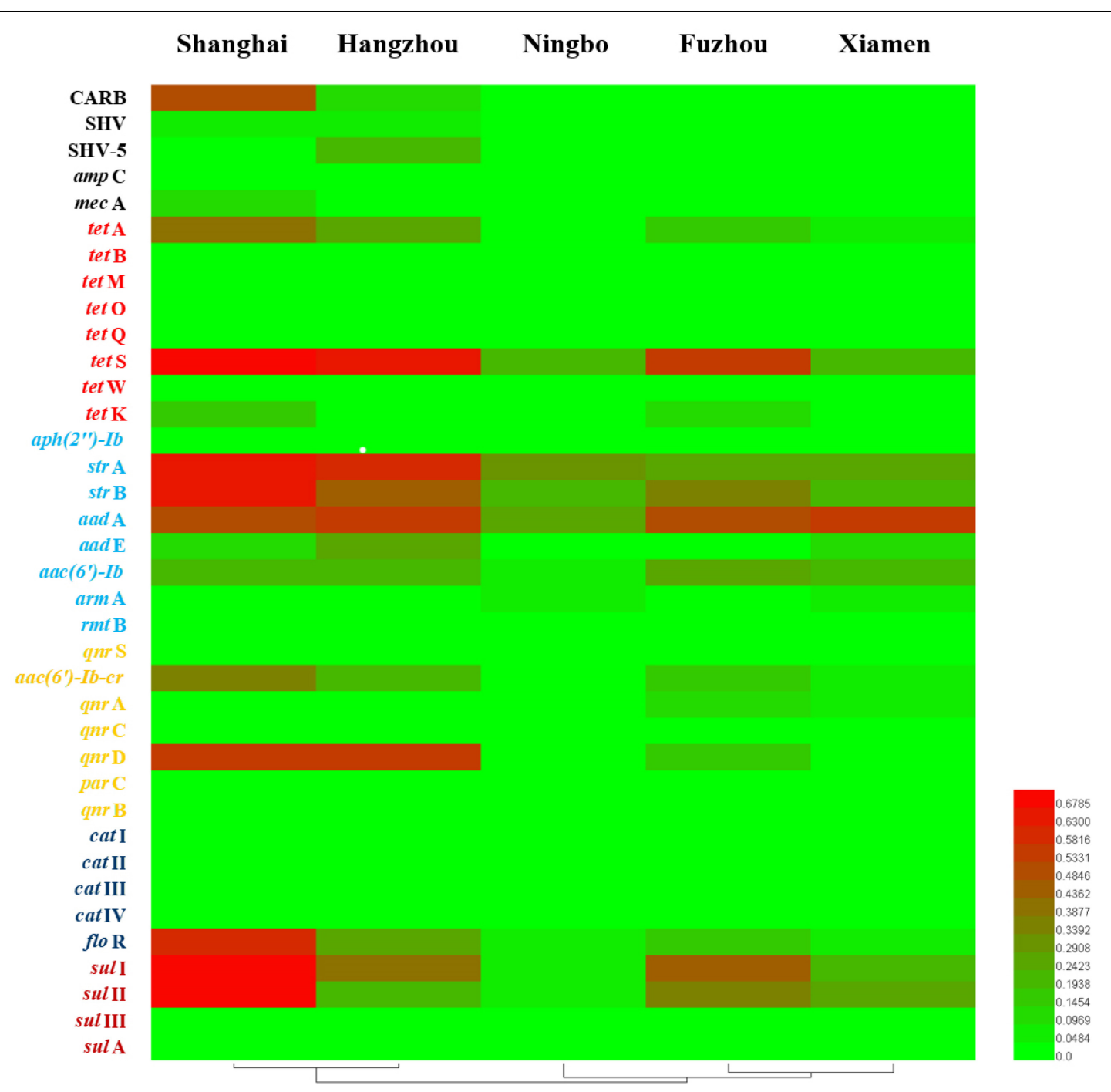

FIGURE 6 | The detection frequencies of 37 ARGs in five southeast coastal cities of China (Shanghai, Hangzhou, Ningbo, Fuzhou, and Xiamen).

TABLE 2 | Relationship between antibiotic residues and ARGs in Shanghai samples.

\begin{tabular}{|c|c|c|c|c|c|c|c|}
\hline \multicolumn{2}{|c|}{ Species } & $\begin{array}{c}\text { Numbers } \\
3\end{array}$ & $\begin{array}{c}\text { Sulfonamides } \\
-\end{array}$ & $\begin{array}{c}\text { Chloramphenicol } \\
-\end{array}$ & $\begin{array}{c}\text { sull } \\
+\end{array}$ & $\begin{array}{c}\text { sull } \\
+\end{array}$ & $\begin{array}{r}\text { floR } \\
+\end{array}$ \\
\hline & Bighead carp (Hypophthalmichthys nobilis) & 1 & - & - & + & + & + \\
\hline & Mandarin fish (Siniperca chuatsi) & 3 & - & - & + & + & + \\
\hline & Crucian carp (Carassius carassius) & 8 & - & - & + & + & + \\
\hline & Snakehead (Channa argus) & 3 & - & - & + & + & + \\
\hline & Grass carp (Ctenopharyngodon idella) & 2 & - & - & - & - & + \\
\hline \multirow[t]{4}{*}{ Marine aquatic animals } & Turbot (Scophthalmus maximus) & 4 & - & - & + & + & + \\
\hline & Large yellow croaker (Larimichthys crocea) & 3 & - & - & + & + & + \\
\hline & Pacific white shrimp (Litopenaeus vannamei) & 1 & - & - & + & + & + \\
\hline & Giant tiger prawn (Penaeus monodon) & 1 & - & - & - & + & - \\
\hline
\end{tabular}

+Detected. - Not detected. 


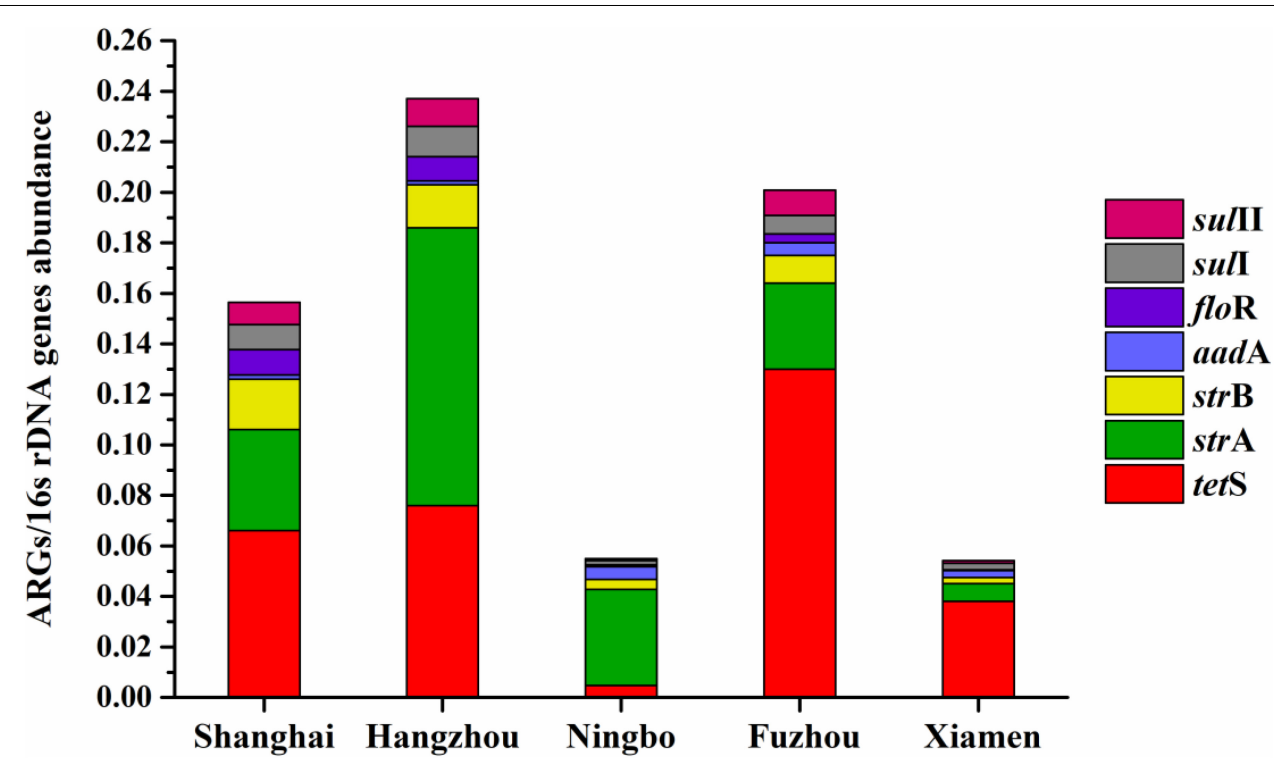

FIGURE 7 | The average relative expression of seven prominent ARGs (tetS, strA, strB, aadA, sull, and sulll) in five southeast coastal cities of China.

receive more antibiotic residues from hospital effluents, plant sewage, urban wastewater and so on. Thus, freshwater animals harbored more ARGs.

We also analyzed the occurrence and abundance of ARGs in five cities. Distinctly, the detection frequencies of ARGs among various geographical locations were different. Shanghai was considered as the most prevalent site with 16 ARGs. Since Shanghai is one of the most urbanized and developed cities in China, and the usage of antibiotics in Shanghai is more than the other four cities (Jiang et al., 2011; Liu et al., 2017). When come to the geographical location differences, anthropogenic activity could address the situation, which might reflect diversity in antibiotic usage in five cities.

The application of antibiotics in aquaculture was one of the important reasons for improving the antibiotics resistance and enhancing the concentration of ARGs in the aquaculture environment (Cesare et al., 2013; Liu et al., 2017). Wide usage of chloramphenicol and sulfonamides in aquaculture has been reported (Li et al., 2006; Heuer et al., 2009; Liu et al., 2017). So, our study simultaneously investigated the concentrations of chloramphenicol and sulfonamides in aquatic animals by HPLCMS/MS. The prominent sulfonamide ARGs, sulI, and sulII were present in aquatic animals but no sulfonamides were detected, this result suggested that sulfonamide-resistance genes sulI and sulII persisted at aquatic animals in the absence of selection pressure. Tetracycline resistance genes and aminoglycoside resistance genes were present at fish farms without presence of the respective antibiotics (Tamminen et al., 2011; Muziasari et al., 2014). The flo $\mathrm{R}$ was the only one chloramphenicol resistance gene detected, but chloramphenicol was not detected in this study, because the Ministry of Agriculture in People's Republic of China banned the use of chloramphenicol in food producing animals in 2002 (No. 193 Bulletin, 2002). Our results suggest that chloramphenicol resistance gene are highly persistent and do not disappear from aquaculture environment, even after several years without chloramphenicol use.

\section{CONCLUSION}

Overall, our study is a first comprehensive research on the occurrences and abundances of 37 ARGs conferring resistance to six classes of antibiotics in skin microbiota of aquatic animals from five cities (Shanghai, Hangzhou, Ningbo, Fuzhou, and Xiamen) located in southeast coastal area of China. Shanghai was considered as the most prevalent site with 16 ARGs, and the geographical location differences could be contributed to anthropogenic activity. The occurrences and abundances of ARGs in freshwater animals were greater than those in marine aquatic animals, which reflect the discrepancy of cultivation pattern between freshwater and marine aquaculture pattern. Interestingly, no target antibiotics were found, sulfonamides resistance genes (sulI, sulII) and chloramphenicol resistance genes (floR) persisted at aquatic animals in the absence of selection pressure. Our study suggests that aquatic animal skin microbiota contribute to the spread of ARGs in water environments, which could provide useful information for better understanding of the contamination caused by ARGs and antibiotics. Results show that ARGs pose an alarmingly serious risk in aquatic animals, which should be paid more attention to by local government.

\section{AUTHOR CONTRIBUTIONS}

$\mathrm{BH}, \mathrm{YB}$, and $\mathrm{LN}$ contributed equally to writing the draft manuscript and carrying out the experiment. FL assisted in completing the experiments. YZ, YP, HL, and ZZ provided support for experimental design and edited the final manuscript. 


\section{FUNDING}

This research was supported by the National Natural Science Foundation of China (31571917 and 31671779), Shanghai Agriculture Applied Technology Development Program (Grant Nos. G20150408, G20160101, and T20170404), Innovation Program of Shanghai Municipal Education Commission (2017-01-07-00-10-E00056), and the "Dawn" Program of Shanghai Education Commission (15SG48).

\section{REFERENCES}

Agersø, Y., and Petersen, A. (2007). The tetracycline resistance determinant tet 39 and the sulphonamide resistance gene sulII are common among resistant Acinetobacter spp. isolated from integrated fish farms in Thailand. J. Antimicrob. Chemother. 59, 23-27. doi: 10.1093/jac/dkl419

Allen, H. K., Donato, J., Wang, H. H., Cloudhansen, K. A., Davies, J., and Handelsman, J. (2010). Call of the wild: antibiotic resistance genes in natural environments. Nat. Rev. Microbiol. 8, 251-259. doi: 10.1038/nrmicro 2312

Amandine, L., Kilunga, P. I., Kayembe, J. M., Naresh, D., Mulaji, C. K., Gregory, G., et al. (2016). Hospital effluents are one of several sources of metal, antibiotic resistance genes, and bacterial markers disseminated in sub-saharan urban rivers. Front. Microbiol. 7:1128. doi: 10.3389/fmicb.2016.01128

Aydin, S., Cetecioglu, Z., Arikan, O., Ince, B., Ozbayram, E. G., and Ince, O. (2015). Inhibitory effects of antibiotic combinations on syntrophic bacteria, homoacetogens and methanogens. Chemosphere 120, 515-520. doi: 10.1016/j. chemosphere.2014.09.045

Bellanger, X., Guilloteau, H., Bonot, S., and Merlin, C. (2014). Demonstrating plasmid-based horizontal gene transfer in complex environmental matrices: a practical approach for a critical review. Sci. Total Environ. 493, 872-882. doi: 10.1016/j.scitotenv.2014.06.070

Cabello, F. C. (2004). Antibiotics and aquaculture in Chile: implications for human and animal health. Rev. Meid. Chil. 132, 1001-1006.

CAP (2016). China Fisheries Statistics. Beijing: China Agricultural Press.

Cesare, A. D., Luna, G. M., Vignaroli, C., Pasquaroli, S., Tota, S., Paroncini, P., et al. (2013). Aquaculture can promote the presence and spread of antibioticresistant Enterococci in marine sediments. PLoS One 8:e62838. doi: 10.1371/ journal.pone. 0062838

Cheesanford, J. C., Aminov, R. I., Krapac, I. J., Garriguesjeanjean, N., and Mackie, R. I. (2001). Occurrence and diversity of tetracycline resistance genes in lagoons and groundwater underlying two swine production facilities. Appl. Environ. Microbiol. 67, 1494-1502. doi: 10.1128/AEM.67.4.1494-1502. 2001

Chen, C. Q., Zheng, L., Zhou, J. L., and Zhao, H. (2017). Persistence and risk of antibiotic residues and antibiotic resistance genes in major mariculture sites in Southeast China. Sci. Total Environ. 580, 1175-1184. doi: 10.1016/j.scitotenv. 2016.12.075

Chen, J., Wei, X. D., Liu, Y. S., Ying, G. G., Liu, S. S., He, L. Y., et al. (2016). Removal of antibiotics and antibiotic resistance genes from domestic sewage by constructed wetlands: optimization of wetland substrates and hydraulic loading. Sci. Total Environ. 565, 240-248. doi: 10.1016/j.scitotenv.2016.04.176

Cheng, W., Chen, H., Su, C., and Yan, S. (2013). Abundance and persistence of antibiotic resistance genes in livestock farms: a comprehensive investigation in eastern China. Environ. Int. 61, 1-7. doi: 10.1016/j.envint.2013.08.023

Cheung, K. C., Leung, H. M., and Wong, M. H. (2008). Metal concentrations of common freshwater and marine fish from the pearl river delta, South China. Arch. Environ. Contam. Toxicol. 54, 705-715. doi: 10.1007/s00244-007-9064-7

Fu, J., Yang, D., Jin, M., Liu, W., Zhao, X., Li, C., et al. (2017). Aquatic animals promote antibiotic resistance gene dissemination in water via conjugation: role of different regions within the zebra fish intestinal tract, and impact on fish intestinal microbiota. Mol. Ecol. 26, 5318-5333. doi: 10.1111/mec.14255

Gao, P., Mao, D., Luo, Y., Wang, L., Xu, B., and Xu, L. (2012). Occurrence of sulfonamide and tetracycline-resistant bacteria and resistance genes in

\section{ACKNOWLEDGMENTS}

We are very grateful to Ben Niu who gave us many advices when we wrote and revised the manuscript.

\section{SUPPLEMENTARY MATERIAL}

The Supplementary Material for this article can be found online at: https://www.frontiersin.org/articles/10.3389/fmicb. 2018.01617/full\#supplementary-material

aquaculture environment. Water Res. 46, 2355-2364. doi: 10.1016/j.watres. 2012.02.004

Gao, Q., Li, Y., Qi, Z., Yue, Y., Min, M., Peng, S., et al. (2018). Diverse and abundant antibiotic resistance genes from mariculture sites of China's coastline. Sci. Total Environ. 630, 117-125. doi: 10.1016/j.scitotenv.2018.02.122

Guglielmetti, E., Korhonen, J. M., Heikkinen, J., Morelli, L., and Wright, A. V. (2010). Transfer of plasmid-mediated resistance to tetracycline in pathogenic bacteria from fish and aquaculture environments. FEMS Microbiol. Lett. 293, 28-34. doi: 10.1111/j.1574-6968.2009.01512.x

Gustafson, R. H., and Bowen, R. E. (1997). Antibiotic use in animal agriculture. J. Appl. Microbiol. 83, 531-541. doi: 10.1046/j.1365-2672.1997.00280.x

Hatha, M., Vivekanandhan, A. A., Joice, G. J., and Christol (2005). Antibiotic resistance pattern of motile aeromonads from farm raised fresh water fish. Int. J. Food Microbiol. 98, 131-134. doi: 10.1016/j.ijfoodmicro.2004.05.017

He, L. Y., Liu, Y. S., Su, H. C., Zhao, J. L., Liu, S. S., Chen, J., et al. (2017). Dissemination of antibiotic resistance genes in representative broiler feedlots environments: identification of indicator ARGs and correlations with environmental variables. Environ. Sci. Technol. 48, 13120-13129. doi: 10.1021/ es5041267

Heuer, O. E., Kruse, H., Grave, K., Collignon, P., Karunasagar, I., and Angulo, F. J. (2009). Human health consequences of use of antimicrobial agents in aquaculture. Clin. Infect. Dis. 49, 1248-1253. doi: 10.1086/605667

Hon, N. T. N., Hoa, T. T. T., Thinh, N. Q., Hinenoya, A., Nakayama, T., Harada, K., et al. (2016). Spread of antibiotic and antimicrobial susceptibility of ESBLproducing Escherichia coli isolated from wild and cultured fish in the mekong delta, vietnam. Fish Pathol. 51, S75-S82. doi: 10.3147/jsfp.51.S75

Ji-Bing, H. E., and Hu, Y. B. (2012). Studies on the pollution levels of antibiotic resistance genes in Jiulong River estuary and wastewater treatment plants in xiamen. Microbiol. China 39, 683-695.

Jiang, L., Hu, X., Yin, D., Zhang, H., and Yu, Z. (2011). Occurrence, distribution and seasonal variation of antibiotics in the Huangpu River, shanghai, China. Chemosphere 82, 822-828. doi: 10.1016/j.chemosphere.2010. 11.028

Khan, G. A., Berglund, B., Khan, K. M., Lindgren, P. E., and Fick, J. (2013). Occurrence and abundance of antibiotics and resistance genes in rivers, canal and near drug formulation facilities - a study in pakistan. PLoS One 8:e62712. doi: 10.1371/journal.pone.0062712

Kümmerer, K., and Henninger, A. (2003). Promoting resistance by the emission of antibiotics from hospitals and households into effluent. Clin. Microbiol. Infect. 9, 1203-1214. doi: 10.1111/j.1469-0691.2003.00739.x

Li, B., Sun, J. Y., Liu, Q. Z., Han, L. Z., Huang, X. H., and Ni, Y. X. (2011). High prevalence of CTX-M $\beta$-lactamases in faecal Escherichia coli strains from healthy humans in Fuzhou, China. Scand. J. Infect. Dis. 43, 170-174. doi: 10. 3109/00365548.2010.538856

Li, H., Tang, R., Lou, Y., Cui, Z., Chen, W., Hong, Q., et al. (2017). A Comprehensive Epidemiological Research for Clinical Vibrio parahaemolyticusin Shanghai. Front. Microbiol. 8:1043. doi: 10.3389/fmicb.2017.01043

Li, X., Hu, Y., Huo, T., and Xu, C. (2006). Comparison of the determination of chloramphenicol residues in aquaculture tissues by time-resolved fluoroimmunoassay and with liquid chromatography and tandem mass spectrometry. Food Agric. Immunol. 17, 191-199. doi: 10.1080/095401006010 90349

Liu, L., Liu, Y. H., Wang, Z., Liu, C. X., Huang, X., and Zhu, G. F. (2014). Behavior of tetracycline and sulfamethazine with corresponding resistance genes from 
swine wastewater in pilot-scale constructed wetlands. J. Hazard. Mater. 278, 304-310. doi: 10.1016/j.jhazmat.2014.06.015

Liu, X., Steele, J. C., and Meng, X. Z. (2017). Usage, residue, and human health risk of antibiotics in Chinese aquaculture: a review. Environ. Pollut. 223, 161-169. doi: 10.1016/j.envpol.2017.01.003

Lou, Y., Liu, H., Zhang, Z., Pan, Y., and Zhao, Y. (2015). Mismatch between antimicrobial resistance phenotype and genotype of pathogenic Vibrio parahaemolyticus isolated from seafood. Food Control 59, 207-211. doi: 10.1016/j.foodcont.2015.04.039

Ma, L., Li, B., and Zhang, T. (2014). Abundant rifampin resistance genes and significant correlations of antibiotic resistance genes and plasmids in various environments revealed by metagenomic analysis. Appl. Microbiol. Biotechnol. 98, 5195-5204. doi: 10.1007/s00253-014-5511-3

Martínez, J. L. (2008). Antibiotics and antibiotic resistance genes in natural environments. Science 321, 365-367. doi: 10.1126/science.1159483

Muziasari, W. I., Managaki, S., Pärnänen, K., Karkman, A., Lyra, C., Tamminen, M., et al. (2014). Sulphonamide and trimethoprim resistance genes persist in sediments at Baltic Sea aquaculture farms but are not detected in the surrounding environment. PLoS One 9:e92702. doi: 10.1371/journal.pone. 0092702

Muziasari, W. I., Pärnänen, K., Johnson, T. A., Lyra, C., Karkman, A., Stedtfeld, R. D., et al. (2016a). Aquaculture changes the profile of antibiotic resistance and mobile genetic element associated genes in Baltic Sea sediments. Fems Microbiol. Ecol. 92:fiw052. doi: 10.1093/femsec/fiw052

Muziasari, W. I., Pitkänen, L. K., Sørum, H., Stedtfeld, R. D., Tiedje, J. M., and Virta, M. (2016b). The resistome of farmed fish feces contributes to the enrichment of antibiotic resistance genes in sediments below Baltic Sea fish farms. Front. Microbiol. 7:2137.

Nakayama, T., Tuyet Hoa, T. T., Harada, K., Warisaya, M., Asayama, M., Hinenoya, A., et al. (2017). Water metagenomic analysis reveals low bacterial diversity and the presence of antimicrobial residues and resistance genes in a river containing wastewater from backyard aquacultures in the Mekong Delta, Vietnam. Environ. Pollut. 222, 294-306. doi: 10.1016/j.envpol.2016. 12.041

No. 193 Bulletin (2002). Ministry of Agriculture of the People's Republic of China. Available at: http://jiuban.moa.gov.cn/zwllm/tzgg/gg/201104/t20110422_ 1976324.htm

Pruden, A., Pei, R., Storteboom, H., and Carlson, K. H. (2006). Antibiotic resistance genes as emerging contaminants: studies in northern colorado. Environ. Sci. Technol. 40, 7445-7450. doi: 10.1021/es0604131

Shen, D. S., Tao, X. Q., Shentu, J. L., and Wang, M. Z. (2014). Residues of veterinary antibiotics in pig feeds and manures in zhejiang province. Adv. Mat. Res. 1010-1012, 301-304. doi: 10.4028/www.scientific.net/AMR.1010-1012.301

Su, H., Liu, S., Hu, X., Xu, X., Xu, W., Xu, Y., et al. (2017). Occurrence and temporal variation of antibiotic resistance genes (ARGs) in shrimp aquaculture: ARGs dissemination from farming source to reared organisms. Sci. Total Environ. 607-608, 357-366. doi: 10.1016/j.scitotenv.2017.07.040

Su, H. C., Pan, C. G., Ying, G. G., Zhao, J. L., Zhou, L. J., Liu, Y. S., et al. (2014). Contamination profiles of antibiotic resistance genes in the sediments at a catchment scale. Sci. Total Environ. 490, 708-714. doi: 10.1016/j.scitotenv.2014. 05.060

Tamminen, M., Karkman, A., Lõhmus, A., Muziasari, W. I., Takasu, H., Wada, S., et al. (2011). Tetracycline resistance genes persist at aquaculture farms in the absence of selection pressure. Environ. Sci. Technol. 45, 386-391. doi: 10.1021/ es $102725 n$
Tang, S., Xiong, J., Wang, K., Hu, C. J., Cheng, K., and Zhang, D. (2017). Dynamic changes of Vibrio community in marine cage culture area of Xiangshan Port. J. Biol. 34, 76-81. doi: 10.3969/j.issn.2095-1736.2017. 04.076

Thomas, C. M., and Nielsen, K. M. (2005). Mechanisms of, and barriers to, horizontal gene transfer between bacteria. Nat. Rev. Microbiol. 3, 711-721. doi: 10.1038/nrmicro1234

Wang, H., Mcentire, J. C., Zhang, L., Li, X., and Doyle, M. (2012). The transfer of antibiotic resistance from food to humans: facts, implications and future directions. Rev. Sci. Tech. 31, 249-260. doi: 10.20506/rst.31.1.2117

Xiong, W., Sun, Y., Zhang, T., Ding, X., Li, Y., Wang, M., et al. (2015). Antibiotics, antibiotic resistance genes, and bacterial community composition in fresh water aquaculture environment in China. Microb. Ecol. 70, 425-432. doi: 10.1007/ s00248-015-0583-x

Xu, W., Zhang, G., Li, X., Zou, S., Li, P., Hu, Z., et al. (2007). Occurrence and elimination of antibiotics at four sewage treatment plants in the Pearl River Delta (PRD), South China. Water Res. 41, 4526-4534. doi: 10.1016/j.watres. 2007.06.023

Yan, H., Zhang, K., Shentu, J., Shen, D., Li, N., and Wang, M. (2018a). Changes to tetracyclines and tetracycline resistance genes in arable soils after single and multiple applications of manure containing tetracyclines. Environ. Sci. Pollut. Res. 25, 5572-5581. doi: 10.1007/s11356-0170853-x

Yan, M., Xu, C., Huang, Y., Nie, H., and Wang, J. (2018b). Tetracyclines, sulfonamides and quinolones and their corresponding resistance genes in the three gorges reservoir, China. Sci. Total Environ. 631-632, 840-848. doi: 10. 1016/j.scitotenv.2018.03.085

Yang, J. F., Ying, G. G., Zhao, J. L., Tao, R., Su, H. C., and Liu, Y. S. (2011). Spatial and seasonal distribution of selected antibiotics in surface waters of the Pearl Rivers, China. J. Environ. Sci. Health B 46, 272-280. doi: 10.1080/03601234. 2011.540540

Ye, K., Wang, H., Zhang, X., Jiang, Y., Xu, X., Zhou, G., et al. (2013). Development and validation of a molecular predictive model to describe; the growth of Listeria monocytogenes in vacuum-packaged chilled pork. Food Control 32, 246-254. doi: 10.1016/j.foodcont.2012.11.017

Zheng, Q., Zhang, R., Wang, Y., Pan, X., Tang, J., and Zhang, G. (2012). Occurrence and distribution of antibiotics in the Beibu Gulf, China: impacts of river discharge and aquaculture activities. Mar. Environ. Res. 78, 26-33. doi: 10.1016/ j.marenvres.2012.03.007

Zhu, Y. G., Johnson, T. A., Su, J. Q., Qiao, M., Guo, G. X., Stedtfeld, R. D., et al. (2013). Diverse and abundant antibiotic resistance genes in Chinese swine farms. Proc. Natl. Acad. Sci. U.S.A. 110, 3435-3440. doi: 10.1073/pnas. 1222743110

Conflict of Interest Statement: The authors declare that the research was conducted in the absence of any commercial or financial relationships that could be construed as a potential conflict of interest.

Copyright (c) 2018 Hong, Ba, Niu, Lou, Zhang, Liu, Pan and Zhao. This is an openaccess article distributed under the terms of the Creative Commons Attribution License (CC BY). The use, distribution or reproduction in other forums is permitted, provided the original author(s) and the copyright owner(s) are credited and that the original publication in this journal is cited, in accordance with accepted academic practice. No use, distribution or reproduction is permitted which does not comply with these terms. 